\title{
http://revistainvestigacionacademicasinfrontera.com
}

\section{LA ACTIVIDAD FÍSICA COMO MEDIDA DE CONTROL DE SOBREPESO Y OBSESIDAD INFANTIL.}

\author{
DRA. GRICELDA HENRY MEJÍA ${ }^{1}$ \\ Universidad de Sonora, Unidad Centro \\ MTRA. MARÍA JULIA LEÓN BAZÁN ${ }^{2}$ \\ Universidad de Sonora, Unidad Centro \\ MTRA. NIDIA CAROLINA BARAHONA HERREJON ${ }^{3}$ \\ Universidad de Sonora, Unidad Centro \\ MTRO. CARLOS ERNESTO OGARRIO PERKINS ${ }^{4}$ \\ Universidad de Sonora, Unidad Centro
}

\footnotetext{
${ }^{1}$ Departamento de Ciencias del Deporte y de la Actividad Física, Universidad de Sonora, Blvd. Luis Encinas y Rosales s/n, Colonia Centro, Hermosillo, Sonora, México Tel. 01(662) 2125936, correo electrónico: grisyhenry@hotmail.com ${ }^{2}$ Departamento de Ciencias del Deporte y de la Actividad Física, Universidad de Sonora, Blvd. Luis Encinas y Rosales s/n, Colonia Centro, Hermosillo, Sonora, México Tel. 01(662)2125936 correo electrónico: julia.leon@unison.mx

${ }^{3}$ Departamento de Ciencias del Deporte y de la Actividad Física, Universidad de Sonora, Blvd. Luis Encinas y Rosales s/n, Colonia Centro, Hermosillo, Sonora, México Tel. 01(662)2125936 correo electrónico: nidiabara67@outlook.com

${ }^{4}$ Departamento de Ciencias del Deporte y de la Actividad Física, Universidad de Sonora, Blvd. Luis Encinas y Rosales s/n, Colonia Centro, Hermosillo, Sonora, México Tel. 01(662)2125936 correo electrónico: ogarrio63@yahoo.com
} 


\title{
http://revistainvestigacionacademicasinfrontera.com
}

\section{RESUMEN}

Este documento presenta los resultados de un proyecto de investigación cuyo objetivo es determinar la importancia de implementar un programa de actividad física basada en el desarrollo de capacidades físicas condicionales y coordinativas a través de ejercicios aeróbicos y anaeróbicos como hábito saludable para el control de sobrepeso y obesidad infantil. Se utilizó un diseño experimental con pre y post prueba, basado en las observaciones de la Organización Mundial de la Salud (OMS) para determinar medidas de peso (Kg) y talla (M) y obtener índice de masa corporal (IMC) que permitiría definir los rangos de sobrepeso y obesidad en los niños. La población consistió en 315 niños y para determinar la muestra, se tomaron medidas de la cintura y pliegue tricipital clasificando a los niños de acuerdo a su estado nutricional considerando las tablas de crecimiento de la OMS y mediante el programa ANTHRO PLUS, resultado una muestra de 41 niños. Posteriormente, en el área de actividad física se aplicó un test y un pre-test, con el apoyo de dos instrumentos: el primero para la medición antropométrica y, el segundo la aplicación de la Batería Eurofit utilizada por Gálvez (2010). Los principales resultados obtenidos mostraron que los niños no bajan ni disminuyen peso porque se aumenta en su talla o estatura. Después de aplicar las pruebas físicas, fue posible verificar que la actividad física es la base fundamental para combatir el sedentarismo y lograr calidad de vida en los niños.

Palabras Clave: Actividad Física, Sobrepeso, Obesidad, Sedentarismo.

\begin{abstract}
This document presents the results of a research project that which aims determine the importance of implementing a program of physical activity based on the development of conditional and coordination physical capacities through aerobic and anaerobic exercises as a healthy habit for the control of overweight and childhood obesity. An experimental design with pre-test and post-test was used, based on the observations of the World Health Organization (WHO) to determine weight (Kg) and height (M) measurements and obtain body mass index (BMI) that would allow define the ranges of overweight and obesity in children. The population consisted of 315 children and to determine the sample, measurements were taken of the waist and triceps fold classifying the children according to their nutritional status considering the WHO growth charts and through the ANTHRO PLUS program, resulting in a sample of 41 children. Subsequently, in the area of physical activity a test and a pre-test were applied, with the support of two instruments: the first for anthropometric measurement and the second the application the Eurofit Battery used by
\end{abstract}




\section{http://revistainvestigacionacademicasinfrontera.com}

Gálvez (2010). The main results obtained showed that children do not lose or lose weight because the size or height increase. After applying the physical tests, it was possible to verify that physical activity is the fundamental basis to combat sedentary lifestyle and achieve quality of life in the children.

Keywords: Physical Activity, Overweight, Obesity, sedentary lifestyle.

\section{Introducción}

En el siglo XXI, los datos de diversos estudios Organización Mundial de la Salud (OMS), Instituto Nacional de Estadística (INE, 2014), muestran un incremento alarmante y muy preocupante de la prevalencia del sobrepeso y de la obesidad, ya no sólo en la población adulta, sino también en la población infantil; siendo un factor de riesgo para producir enfermedades que supone un aumento de los costos sanitarios, al igual que empeora la calidad de vida en las personas adultas.

El incremento tan abrupto de la obesidad que ha ocurrido en las últimas décadas, así como su gran extensión, obedece principalmente a cambios importantes en la alimentación de la población, al patrón de actividad física y a otros factores de índole sociocultural como son los medios de comunicación (Peña y Bacallao, 2001; Figueroa, 2009). Por otro lado, el excesivo consumo de alimentos de alta densidad energética, ricos en grasas saturadas, azúcar y sal, la falta de actividad física y el incremento de actividades sedentarias, representan en la actualidad los estilos de vida de gran parte de la población infantil (Kipping, Jago y Lawlor 2008; Moreno et al., 2010).

Este desequilibrio entre la ingesta y el gasto energético sostenido por períodos prolongados de tiempo, ha sido reconocido internacionalmente como la principal causa de la elevada y creciente 


\section{http://revistainvestigacionacademicasinfrontera.com}

prevalencia de obesidad (Rodríguez et al., 2012). Actualmente existen diversas causas del incremento de la obesidad infantil en México; entre otros, los factores más relevantes son los medios de comunicación, la falta de actividad física en la población, los hábitos alimenticios no son los correctos y en combinación con la facilidad con la que se puede consumir comida chatarra (Mercado y Vilchis, 2013).

Las evidencias que relacionan la escasa o nula práctica de actividad física con la prevalencia de esas enfermedades aumentan rápidamente y reflejan la importancia que está adquiriendo la actividad física como determinante de salud en los niños (Rodríguez et al., 2016). El sedentarismo es la ausencia de actividad física necesaria para que el organismo se mantenga en un estado saludable y, es una problemática que provoca sobrepeso y obesidad en la población infantil, la escuela parece ser un lugar favorable a la hora de orientar la conducta del niño mediante estilos de vida saludables y prevenir la obesidad y sus enfermedades asociadas (OMS, 2016).

La adopción de un estilo de vida saludable basado en la actividad física ayuda a disminuir sobrepeso y la obesidad en la población infantil. Este documento presenta los resultados de un proyecto de investigación que tiene como objetivo determinar la importancia de implementar un programa de actividad física basada en el desarrollo de capacidades físicas condicionales y coordinativas a través de ejercicios aeróbicos y anaeróbicos como hábito saludable para el control de sobrepeso y obesidad infantil.

\section{Marco Teórico-Conceptual}

\subsection{Situación actual del sobrepeso y obesidad en niños en México}

Onis, Blössner y Borghi (2010) estimaban que, en todo el mundo, tenían sobrepeso o estaban obesos 43 millones de niños con edades inferiores a los cinco años. De ellos, 35 millones vivían en países en desarrollo. Los mismos autores indican que el número de niños con sobrepeso 


\section{http://revistainvestigacionacademicasinfrontera.com}

y obesidad se incrementará a 60 millones en el año 2020. Teniendo en cuenta esta cifra, la prevención es una parte vital del esfuerzo para contener esta marea creciente de obesidad en la niñez, habiendo alcanzado dimensiones epidémicas. El sobrepeso y la obesidad se encuentran en el quinto riesgo principal de mortalidad (OMS, 2009).

Datos de la OMS indican que desde el año 1980 la obesidad ha aumentado a más del doble en todo el mundo. En el año 2008, 1.500 millones de adultos tenían exceso de peso. Dentro de este grupo, más de 200 millones de hombres y cerca de 300 millones de mujeres eran obesos, por lo cual la OMS ha declarado a la obesidad y al sobrepeso con el carácter de epidemia mundial. Representa además una gran carga económica para los presupuestos destinados a la salud, por sus elevados costos asociados tanto directos como indirectos. Se estima que tanto el sobrepeso como la obesidad son responsables del $44 \%$ de diabetes, del 23\% de cardiopatías isquémicas y entre el 7 y el 41\% de algunos cánceres (Moreno, 2012).

La clasificación internacional actual de obsesidad para un adulto es la propuesta por la OMS y, está basada en el Índice de Masa Corporal (IMC), el cual corresponde a la relación entre el peso expresado en kilos y el cuadrado de la altura, expresada en metros. De esta manera, las personas cuyo cálculo de IMC sea igual o superior a $30 \mathrm{~kg} / \mathrm{m} 2$ se consideran obesas (Tabla 1).

Tabla 1. Clasificación de la obesidad según la OMS

\begin{tabular}{|l|l|l|}
\hline Clasificación & $\mathbf{M C}\left(\mathbf{k g} / \mathbf{m}^{\mathbf{2}}\right)$ & Riesgo Asociado a la salud \\
\hline Normo Peso & $18.5-24.9$ & Promedio \\
Exceso de Peso & 25 & \\
Sobrepeso o Pre Obeso & $25-29.9$ & AUMENTADO \\
Obesidad Grado l o moderada & $30-34.9$ & AUMENTO MODERADO \\
Obesidad Grado ll o severa & $35-39.9$ & AUMENTO SEVERO \\
Obesidad Grado Ill o mórbida & $\geq 40$ & AUMENTO MUY SEVERO \\
\hline
\end{tabular}

Fuente: Organización Mundial de Salud. 


\section{http://revistainvestigacionacademicasinfrontera.com}

Según la definición de obesidad, las personas obesas son aquellas que tienen un exceso de grasa corporal. Se considera que un hombre adulto de peso normal presenta un contenido de grasa en un rango del 15 al 20\% del peso corporal total, y las mujeres entre el 25 y $30 \%$ del peso corporal total. Deurenberg y colaboradores establecieron una ecuación para estimar el porcentaje total de grasa en adultos, basado en el IMC, la edad y el sexo.

Sin embargo, en el caso de los niños, el IMC varía de manera importante a través de los años, presentando un aumento sustancial en el primer año de vida. Posteriormente disminuye hasta alrededor de los 5 a 6 años de edad. Luego se observa nuevamente un aumento del IMC conocido como "rebote adipocitario". En niños mayores de 6 años, la obesidad se define basada en curvas de crecimiento según sexo, IMC y edad. La Organización Mundial de la Salud presenta la clasificación de cómo deben interpretarse para niños y niñas los percentiles de IMC según edad y sexo (Tabla 2).

Tabla 2. IMC para niños y niñas 
Núm. 26

\section{http://revistainvestigacionacademicasinfrontera.com}
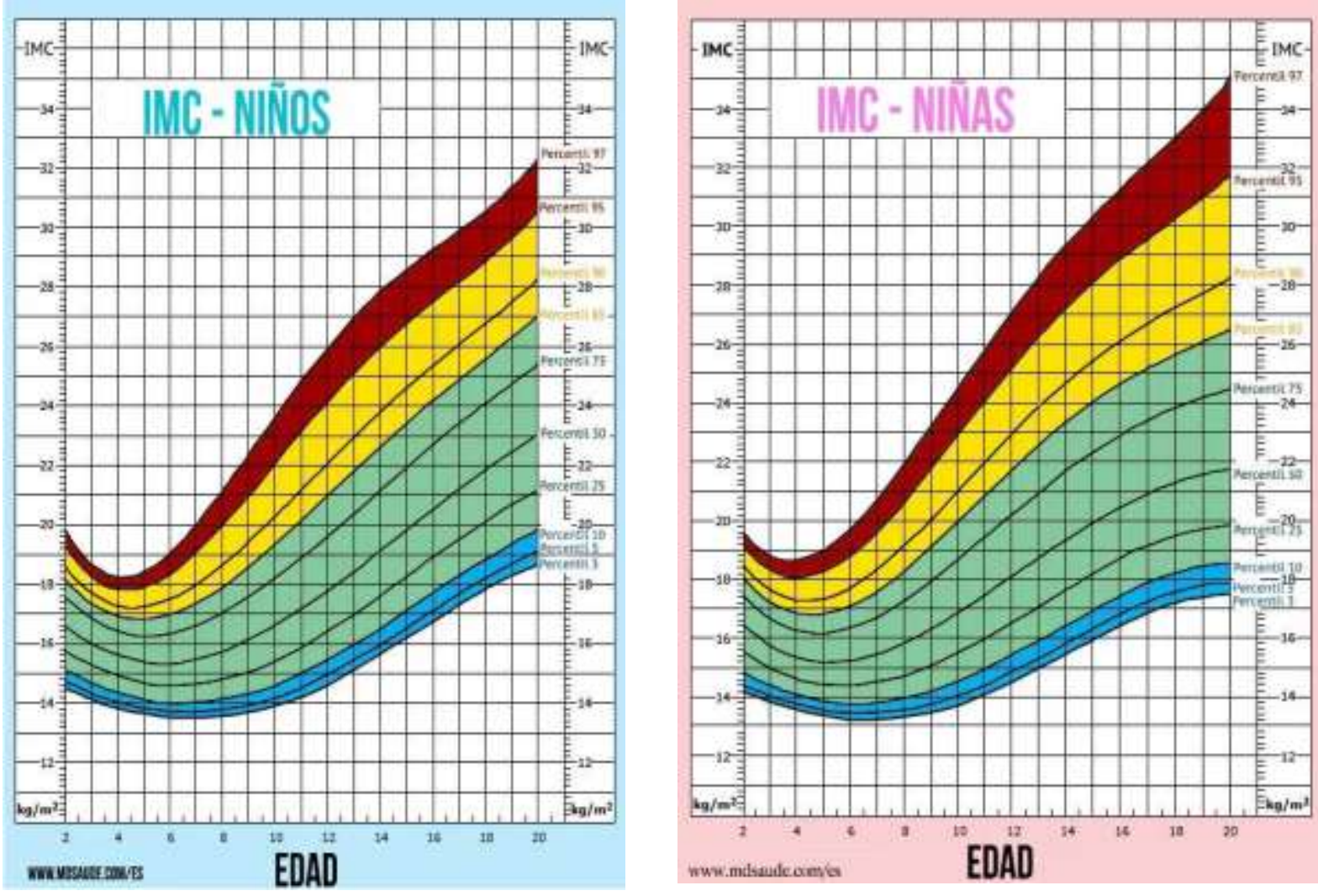

Fuente: Organización Mundial de la Salud.

La obesidad es una problemática que concierna a cualquier país y sobre todo a los países en desarrollo como México. Según señalan datos sobre obesidad infantil, ha habido un aumento dramático en los pasados diez años. En México, las encuestas nacionales de salud de los años 1999 y 2006 demuestran que tanto el sobrepeso como la obesidad en niños de entre 5 y 11 años aumentaron casi un 40\%. La Encuesta Nacional de Salud y Nutrición (ENSANUT, 2012) reveló que en la zona norte de México tiene la tasa más alta de sobrepeso y obesidad en adultos y, en particular, Sonora ocupó el primer lugar con $74.7 \%$ de adultos con este padecimiento. La encuesta indicó que el $70.6 \%$ de los hombres y el $76.9 \%$ de las mujeres mayores de 20 años padecen obesidad; así como en el 36.9\% de los niños entre cinco y 11 años y en el 35.2\% en los adolescentes de 12 a 19 años. 


\section{http://revistainvestigacionacademicasinfrontera.com}

Posteriormente ENSANUT (2016), realiza la misma encuesta donde evaluó la prevalencia de sobrepeso y obesidad en niños y adolescentes y, los resultados variaron según sexo (masculino o femenino) y lugar de residencia (zona rural o urbana). En los resultados de la encuesta se muestra que el sobrepeso y la obesidad en mujeres presenta un aumento respecto a cifras de 2012, el cual es mayor en zonas rurales que urbanas y, en referencia a los niños y adolescentes los principales resultados se presentan a continuación:

1. NIÑOS EN EDAD ESCOLAR - 5 a 11 años de edad

- Tres de cada 10 menores padecen sobrepeso u obesidad (prevalencia combinada de 33.2\%). En 2012, la prevalencia en este grupo de edad era de 34.4\%. En esta última medición se observa una disminución significativa del sobrepeso en niños varones.

- Se observa un incremento progresivo en la prevalencia combinada de sobrepeso y obesidad en zonas rurales en ambos sexos.

2. ADOLESCENTES - 12 a 19 años de edad

- Casi 4 de cada 10 adolescentes presenta sobrepeso u obesidad (prevalencia combinada de $36.3 \%$ ). En 2012, esta cifra era de $34.9 \%$.

- En mujeres adolescentes, se observó un aumento del 2.7 puntos porcentuales en sobrepeso, alcanzando un nivel de $26.4 \%$. La prevalencia combinada de sobrepeso y obesidad en mujeres adolescentes fue de $39.2 \%$

- En hombres adolescentes se presenta una reducción, de $34.1 \%$ a $33.5 \%$ en prevalencia combinada.

Para Ceballos, Álvarez, Torres y Zaragoza (2009), existen dos mayores consecuencias de la obesidad infantil. La primera está relacionada con cambios psicológicos. Ellos ocurren 


\section{http://revistainvestigacionacademicasinfrontera.com}

temprano en la vida e incluyen: baja autoestima, bajos resultados en la escuela, un cambio en la auto-imagen, particularmente durante la adolescencia, introversión, a menudo seguida de rechazo social. La segunda consecuencia guarda relación con el aumento del riesgo de desarrollar enfermedades o condiciones patológicas. Que generalmente pueden desarrollarse en la edad adulta, pero que cada vez se diagnostican a menor edad.

\subsection{La importancia de la Actividad física para el control y prevención de la obesidad y sobrepeso infantil}

La Actividad Física se define como cualquier movimiento corporal realizado por el sistema músculo-esquelético y que provoca un gasto de energía superior al nivel basal, como por ejemplo caminar, subir y bajar escaleras, levantar objetos, barrer o limpiar entre otros. Estas actividades, son consideradas actividades de tipo aeróbico, en las cuales se emplean gran cantidad de músculos y de energía, favoreciendo el aumento de la frecuencia cardíaca y respiratoria, así como la capacidad de transporte del oxígeno celular (Sallis y Owen, 1999).

La actividad física abarca el ejercicio, pero también otras actividades que entrañan movimiento corporal y se realizan como parte de los momentos de juego, del trabajo, de formas de transporte activas, de las tareas domésticas y de actividades recreativas. Así pues, resaltando la diferencia de conceptos y tal como nos explica Garita (2006), actividad física se conoce como todo aquel movimiento que realiza el ser humano que implica el desplazamiento de los diversos componentes corporales y el gasto energético que pueden ser realizados durante el diario vivir, como ocupación, distracción, ejercicio y deporte. El ejercicio, por su parte, es un tipo de actividad física, planeada y estructurada, en la que el hombre participa con el objetivo establecido de mejorar alguna de sus cualidades físicas como la fuerza, la potencia, la velocidad, la resistencia aeróbica, 
Núm. 26

ISSN: 2007-8870

\section{http://revistainvestigacionacademicasinfrontera.com}

entre otras; y el deporte, es la actividad física organizada en ligas con competición individual o grupal (Nigg, 2003)

Existe una clara relación entre el nivel de actividad física durante la infancia y la adolescencia y la condición de salud en la edad adulta. En México, existen diversas causas del incremento de la obesidad infantil, en primer lugar, contribuye a controlar y prevenir algunas enfermedades como la obesidad y la diabetes tipo 2 en los niños y adolescentes, mejorando la sensibilidad de los receptores de membrana por la adrenalina y la insulina, hormonas encargadas del metabolismo de los carbohidratos; se ha demostrado que la práctica de Actividad Física regular y especialmente el ejercicio de tipo aeróbico y de resistencia, contribuyen a disminuir el riesgo de diabetes tipo 2 en un 95\%, cuando el gasto de energía por semana es superior a $500 \mathrm{Kcal}$, este beneficio fue observado especialmente en la población joven con valores altos (percentil $\geq 85$ ) de índice de masa corporal (IMC) (Bloomgarden, 2004).

En segundo lugar, aunque las enfermedades cardiovasculares no se manifiestan en los niños, los malos hábitos desde esta edad pueden ser corregidos con el fin de fomentar y mantener aquellos que sean positivos para la salud y la vida, contribuyendo así a disminuir el deterioro de la calidad de vida en la adultez; se ha demostrado que la práctica de Actividad Física previene la mortalidad en la edad adulta, particularmente secundaria a enfermedades cardiovasculares, la hipertensión, la hipercolesterolemia y algunos tipos de cáncer (Nielsen y Andersen, 2003; Strong et al., 2005; Pearson, Manor y Power, 2006; Mercado, 2013).

Existen muchas y diversas razones para justificar la implementación de políticas públicas orientadas a incrementar el número de ciudadanos físicamente activos. Ya sea por cuestiones de salud, laborales, económicas o sociales, en países como Escocia (Eberth y Smith, 2010), España (Lera, García, y Suárez, 2009), Canadá (Humphreys y Ruseski, 2009), Australia (Brown y Roberts, 2010) y el Reino Unido (Downward y Riordan, 2007; Kwame, 2009), la temática del ejercicio se ha establecido como prioridad en la agenda de política pública. México no es la excepción, su 


\section{http://revistainvestigacionacademicasinfrontera.com}

Programa Nacional de Cultura Física y Deporte plantea la meta de pasar de 5.1 millones de personas que participaban en actividades físicas regularmente en 2006, a treinta y cinco millones en 2012.

En noviembre del 2013, el Instituto Nacional de Estadística y Geografía INEGI en conjunto con la Comisión Nacional del Deporte (Conade), mencionan que los resultados del estudio titulado Módulo de Práctica deportiva y ejercicio físico, el $56.2 \%$ de los mexicanos mayores de 18 años no hace ninguna actividad física y el 18\% nunca ha practicado una actividad física en su tiempo libre. Por su parte, el INEGI da a conocer los resultados del Módulo de Práctica Deportiva y Ejercicio Físico (MOPRADEF), correspondientes a noviembre de 2016 revela que $58.2 \%$ de la población mexicana de 18 y más años es inactiva físicamente.

El sobrepeso y la obesidad, así como sus enfermedades no transmisibles asociadas, son en gran parte prevenibles. Barlow y Dietz (2009), mencionan que para apoyar a los niños y jóvenes en el proceso de realizar elecciones, en materia de alimentos y actividad física periódica, y en consecuencia prevenir la obesidad, es fundamental: limitar la ingesta energética procedente de la cantidad de grasa total; aumentar el consumo de frutas y verduras, así como de leguminosas, cereales integrales y frutos secos; limitar la ingesta de azúcares; realizar una actividad física periódica, y lograr un equilibrio energético y un peso normal.

El hecho de que los niños, durante todo el proceso de crecimiento, tiendan a estar en actividad no es un hecho casual. El ejercicio no es una actividad que influya solamente en el mantenimiento del peso o en el desarrollo muscular, sino que realmente es un estímulo que potencia el desarrollo y la funcionalidad de la mayoría de nuestros órganos. Los ejercicios de psicomotricidad, por ejemplo, cuando se realizan adecuadamente desde los primeros meses de vida, 


\section{http://revistainvestigacionacademicasinfrontera.com}

son un magnífico estímulo para el desarrollo de las funciones cerebrales (Ramírez, Vinaccia y Suárez, 2004). Y lo que es más importante, la infancia y la adolescencia son los periodos ideales para introducir el aprendizaje de los hábitos de vida que resultarán más productivos y saludables durante la vida adulta. Los estudios muestran que cuanto antes se incorpore la actividad física como hábito en la infancia, mayores son las probabilidades de que éste se mantenga en la edad adulta (Eberth y Smith, 2010; Mercado, 2013).

El bajo tiempo dedicado a la actividad física, el exagerado tiempo dedicado a las actividades sedentarias o los malos hábitos alimentarios son conductas que se están adoptando en gran parte de la población infantil occidental (Elena, 2006; Rodríguez, 2006; Crovetto, Zamorano y Medinelli, 2010; Bucco y Zubiar, 2013). Los niños con sobrepeso y obesidad practican menos actividad física y más actividades sedentarias. Los cambios en los hábitos de actividad física en los niños y las presiones académicas por obtener mayor rendimiento se han planteado como los principales responsables del sedentarismo infantil. Sumado a los cambios en los hábitos alimentarios, algunas características conductuales, emotivas y cognitivas detectadas en los niños sedentarios han llevado a aumentar considerablemente los niveles sobrepeso/obesidad infantil en la actualidad.

De acuerdo con el Instituto Nacional de Salud Pública, la obesidad es una enfermedad crónica, compleja y multifactorial que puede prevenirse. Una de las principales causas de la obesidad en los niños y jóvenes mexicanos es el sedentarismo, debido a la disminución del tiempo que dedican a realizar actividades físicas; pasan gran parte de su tiempo frente a una computadora, viendo televisión o jugando video-juegos (Salud, 2012).

\section{Diseño de la investigación}

Se utilizó un diseño experimental con pre-prueba y post-prueba, tomando como base las observaciones de la Organización Mundial de la Salud, donde menciona la importancia de desarrollar actividad física recreativa y deportiva de lunes a viernes con 60 minutos diarios con 


\section{http://revistainvestigacionacademicasinfrontera.com}

intensidad de moderada a vigorosa, propiciando que los participantes gasten las calorías que ingieren. Este programa se realizó del 11 de septiembre del 2016 al 19 de junio del 2017, con la autorización de las autoridades correspondientes de la escuela, así como también el consentimiento de los padres de familia, de lunes a viernes de 13:00 a 14:00 hrs. El estudio se llevó a cabo en el Colegio José Lafountaine, en Hermosillo, Sonora debido a que es una escuela de tiempo completo. La institución cuenta con una población de 315 niños con edades comprendidas entre 9 y 12 años, de los cuales 166 son mujeres y 149 hombres.

Para la determinación de la muestra se tomó como base que los alumnos participaran en forma voluntaria, permiso de los padres de familia, contaran con las medidas de peso $(\mathrm{Kg})$ y talla (M) que se realizaron con un estadiómetro, balanza, cinta y plicómetro; marca Seca, para obtener el índice de masa corporal (IMC) que marca la OMS. Definiendo con los datos el índice de masa corporal en los rangos de sobrepeso u obesidad, así como también se tomaron medidas de la cintura y pliegue tricipital clasificando a los niños de acuerdo a su estado nutricional considerando las tablas de crecimiento de la OMS y mediante el programa ANTHRO PLUS, dando como resultado una muestra de 41 niños. Posteriormente, en el área de actividad física se aplicó un test y un pretest, con el apoyo de dos instrumentos: el primero para la medición antropometrica y, el segundo instrumento fue la aplicación de la Batería Eurofit utilizada por Gálvez (2010), para conocer el estado inmedito de la aptitud física del alumno, así como sus cambios y tendencias mediante una serie de test relevantes y experimentados de equilibrio, flexibilidad, abdominales, salto de longitud sin carrera, velocidad y resistencia con course navette de 20 metros.

Las actividades realizadas fueron de tipo aeróbicas, que son las más indicadas para combatir el sobrepeso y la obesidad. En todas las sesiones se inicia con una preparación fisiológica del organismo, una parte principal donde se desarrolló el $80 \%$ de las actividades y por último una vuelta a la calma. Entre las actividades que se realizaron se incluyen las siguientes: Juegos 
Núm. 26

ISSN: 2007-8870

\section{http://revistainvestigacionacademicasinfrontera.com}

colectivos: serán de tipo aeróbico y con mucho carácter lúdico, deportes colectivos: fútbol, voleibol, baloncesto y recreación.

\section{Presentación, análisis e interpretación de resultados}

Se presentan a continuación los resultados más relevantes basados en el objetivo central de esta investigación. En la Tabla 3 se muestran las medidas antropométricas al inicio y al final de la investigación donde se tomaron en cuenta las medidas de talla, peso, circunferencia, pliegue tricipital, IMC y percentil para determinar la clasificación de los niños en el grado de sobrepeso y obesidad, los resultados muestran que al final de la prueba comparada con el inicio del total de los niños solo seis modificaron su clasificación del peso de obesidad a sobrepeso y, el peso obtenido en los resultados se debe a que aumentaron su peso en relación al crecimiento.

Tabla 3; Medidas Antropométricas

\begin{tabular}{|c|c|c|c|c|c|c|c|c|c|c|c|c|}
\hline No. & Sexo & $\begin{array}{c}\text { Fecha de } \\
\text { nacimiento }\end{array}$ & Edad & Fechas & $\begin{array}{c}\text { Periodo } \\
\text { Medición }\end{array}$ & Talla & Peso & Circunferencia & Tricipital & IMC & Percentil & Clasificación \\
\hline 1 & $\mathbf{0}$ & $16 / 08 / 07$ & 9 & $11 / 11 / 16$ & Inicial & 147 & 57.9 & 90.6 & 20 & 26.8 & 98 & obesidad \\
\hline & & & 10 & $19 / 06 / 17$ & Final & 149.5 & 59.6 & 81.5 & 23 & 26.7 & 98 & obesidad \\
\hline 2 & $\mathbf{0}$ & $21 / 11 / 07$ & 8 & $11 / 11 / 16$ & Inicial & 139 & 55.3 & 87.2 & 23 & 28.6 & $99+$ & obesidad \\
\hline & & & 9 & $19 / 06 / 17$ & Final & 142 & 58 & 90 & 21 & 28.8 & $99+$ & obesidad \\
\hline 3 & $\mathbf{0}$ & $31 / 01 / 07$ & 9 & $11 / 11 / 16$ & Inicial & 140 & 49 & 76.3 & 20 & 25 & 97 & obesidad \\
\hline & & & 10 & $19 / 06 / 17$ & Final & 145 & 51.5 & 73.5 & 20 & 24.5 & 96 & obesidad \\
\hline 4 & $\mathbf{1}$ & $10 / 08 / 07$ & 9 & $11 / 11 / 16$ & Inicial & 145 & 53.4 & 83.4 & 19 & 25.4 & 98 & obesidad \\
\hline & & & 10 & $19 / 06 / 17$ & Final & 147 & 57.1 & 85 & 17.5 & 26.4 & 98 & obesidad \\
\hline 5 & $\mathbf{1}$ & $11 / 12 / 07$ & 8 & $11 / 11 / 16$ & Inicial & 137 & 49.9 & 80.0 & 13 & 26.6 & 99 & obesidad \\
\hline & & & 9 & $19 / 06 / 17$ & Final & 141.5 & 51.7 & 76 & 16.5 & 25.8 & 98 & obesidad \\
\hline 6 & $\mathbf{0}$ & $23 / 06 / 07$ & 9 & $11 / 11 / 16$ & Inicial & 140 & 41.2 & 87.3 & 18 & 21 & 91 & sobrepeso \\
\hline & & & 10 & $19 / 06 / 17$ & Final & 144.4 & 45 & 78 & 18 & 21.6 & 91 & sobrepeso \\
\hline 7 & $\mathbf{0}$ & $07 / 08 / 07$ & 9 & $11 / 11 / 16$ & Inicial & 135 & 46.5 & 83.2 & 22 & 25.5 & 98 & obesidad \\
\hline & & & 10 & $19 / 06 / 17$ & Final & 140 & 49 & 77 & 21.5 & 25 & 97 & obesidad \\
\hline 8 & $\mathbf{1}$ & $06 / 07 / 07$ & 9 & $11 / 11 / 16$ & Inicial & 137 & 44.4 & 76.3 & 18 & 23.7 & 97 & obesidad \\
\hline & & & 10 & $19 / 06 / 17$ & Final & 141 & 48.9 & 75 & 15 & 24.6 & 97 & obesidad \\
\hline
\end{tabular}


http://revistainvestigacionacademicasinfrontera.com

\begin{tabular}{|c|c|c|c|c|c|c|c|c|c|c|c|c|}
\hline 9 & 0 & 03/08/07 & 9 & $11 / 11 / 16$ & Inicial & 131 & 38.3 & 75.6 & 17 & 22.3 & 95 & obesidad \\
\hline & & & 10 & $19 / 06 / 17$ & Final & 135 & 42.2 & 78 & 18 & 23.2 & 97 & obesidad \\
\hline \multirow[t]{2}{*}{10} & 1 & 09/11/07 & 8 & $11 / 11 / 16$ & Inicial & 133 & 41.9 & 77.2 & 21 & 23.7 & 97 & obesidad \\
\hline & & & 9 & $19 / 06 / 17$ & Final & 138 & 45.7 & 77 & 22 & 24 & 97 & obesidad \\
\hline \multirow[t]{2}{*}{11} & 0 & $24 / 04 / 07$ & 9 & $11 / 11 / 16$ & Inicial & 132 & 37.2 & 71.0 & 12 & 21.3 & 92 & sobrepeso \\
\hline & & & 10 & $19 / 06 / 17$ & Final & 135 & 39.7 & 67 & 14 & 21.8 & 92 & sobrepeso \\
\hline \multirow[t]{2}{*}{12} & 1 & $24 / 09 / 06$ & 10 & $11 / 11 / 16$ & Inicial & 140 & 52 & 92.0 & 21 & 26.5 & 98 & obesidad \\
\hline & & & 11 & $19 / 06 / 17$ & Final & 144 & 56 & 93 & 20 & 27 & 98 & obesidad \\
\hline \multirow[t]{2}{*}{13} & 1 & $18 / 06 / 06$ & 10 & $11 / 11 / 16$ & Inicial & 142 & 50.2 & 79.1 & 16 & 24.9 & 97 & obesidad \\
\hline & & & 11 & $19 / 06 / 17$ & Final & 146.5 & 54.9 & 84 & 16.5 & 25.6 & 97 & obesidad \\
\hline \multirow[t]{2}{*}{14} & 1 & $10 / 02 / 06$ & 10 & $11 / 11 / 16$ & Inicial & 144 & 52 & 89.5 & 24 & 25.1 & 97 & obesidad \\
\hline & & & 11 & $19 / 06 / 17$ & Final & 149 & 52.2 & 90 & 23.5 & 23.5 & 94 & sobrepeso \\
\hline \multirow[t]{2}{*}{15} & $\mathbf{0}$ & 09/01/06 & 10 & $11 / 11 / 16$ & Inicial & 148 & 52.9 & 78.0 & 20 & 24.2 & 95 & obesidad \\
\hline & & & 11 & $19 / 06 / 17$ & Final & 153 & 54 & 70 & 16.5 & 23.1 & 92 & sobrepeso \\
\hline \multirow[t]{2}{*}{16} & $\mathbf{0}$ & $25 / 10 / 06$ & 10 & $11 / 11 / 16$ & Inicial & 146 & 51.7 & 72.0 & 21 & 24.3 & 96 & obesidad \\
\hline & & & 11 & $19 / 06 / 17$ & Final & 149.5 & 56 & 72 & 24 & 25.1 & 96 & obesidad \\
\hline \multirow[t]{2}{*}{17} & $\mathbf{0}$ & $12 / 07 / 06$ & 10 & $11 / 11 / 16$ & Inicial & 138 & 46.1 & 78.4 & 23 & 24.2 & 96 & obesidad \\
\hline & & & 11 & $19 / 06 / 17$ & Final & 142.5 & 50 & 79 & 21 & 24.6 & 95 & obesidad \\
\hline \multirow[t]{2}{*}{18} & $\mathbf{0}$ & $13 / 09 / 06$ & 10 & $11 / 11 / 16$ & Inicial & 155 & 60.7 & 88.0 & 24 & 25.3 & 97 & obesidad \\
\hline & & & 11 & $19 / 06 / 17$ & Final & 161 & 65.9 & 78.5 & 26 & 25.4 & 96 & obesidad \\
\hline \multirow[t]{2}{*}{19} & $\mathbf{0}$ & $26 / 02 / 06$ & 10 & $11 / 11 / 16$ & Inicial & 144 & 55.8 & 87.8 & 18 & 26.9 & 97 & obesidad \\
\hline & & & 11 & $19 / 06 / 17$ & Final & 147 & 59.9 & 93.5 & 21 & 27.7 & 97 & obesidad \\
\hline \multirow[t]{2}{*}{20} & 0 & 23/07/06 & 10 & $11 / 11 / 16$ & Inicial & 162 & 64.8 & 81.1 & 23 & 24.7 & 96 & obesidad \\
\hline & & & 11 & $19 / 06 / 17$ & Final & 168 & 67.3 & 76 & 21 & 23.8 & 94 & sobrepeso \\
\hline \multirow[t]{2}{*}{21} & 0 & $10 / 05 / 06$ & 10 & $11 / 11 / 16$ & Inicial & 144 & 55.8 & 93.2 & 22 & 26.9 & 98 & obesidad \\
\hline & & & 11 & $19 / 06 / 17$ & Final & 149 & 58 & 84 & 23 & 26.1 & 97 & obesidad \\
\hline \multirow[t]{2}{*}{22} & 1 & $12 / 02 / 06$ & 10 & $11 / 11 / 16$ & Inicial & 153.5 & 52.3 & 91.5 & 16 & 22.2 & 95 & obesidad \\
\hline & & & 11 & $19 / 06 / 17$ & Final & 157.5 & 50.8 & 78 & 16 & 20.5 & 88 & sobrepeso \\
\hline \multirow[t]{2}{*}{23} & 1 & $15 / 09 / 06$ & 10 & $11 / 11 / 16$ & Inicial & 143 & 48 & 81.5 & 21 & 23.5 & 96 & obesidad \\
\hline & & & 11 & $19 / 06 / 17$ & Final & 147.5 & 52.7 & 77.5 & 20 & 24.2 & 96 & obesidad \\
\hline \multirow[t]{2}{*}{24} & 0 & $21 / 10 / 06$ & 10 & $11 / 11 / 16$ & Inicial & 153 & 69.2 & 92 & 25 & 29.6 & $99+$ & obesidad \\
\hline & & & 11 & $19 / 06 / 17$ & Final & 157.5 & 71.1 & 86 & 25 & 28.7 & 98 & obesidad \\
\hline \multirow[t]{2}{*}{25} & 0 & 09/08/06 & 10 & $11 / 11 / 16$ & Inicial & 141.5 & 50.3 & 84.7 & 21 & 25.1 & 97 & obesidad \\
\hline & & & 11 & $19 / 06 / 17$ & Final & 146 & 51.5 & 76 & 24 & 24.2 & 95 & obesidad \\
\hline \multirow[t]{2}{*}{26} & 1 & $02 / 03 / 06$ & 10 & $11 / 11 / 16$ & Inicial & 132 & 50.1 & 82.5 & 24 & 28.8 & 98 & obesidad \\
\hline & & & 11 & $19 / 06 / 17$ & Final & 142.5 & 57.1 & 84 & 31 & 28.1 & 98 & obesidad \\
\hline 27 & $\mathbf{0}$ & 04/08/06 & 10 & $11 / 11 / 16$ & Inicial & 145 & 47.6 & 78 & 15 & 22.6 & 93 & sobrepeso \\
\hline
\end{tabular}




\section{http://revistainvestigacionacademicasinfrontera.com}

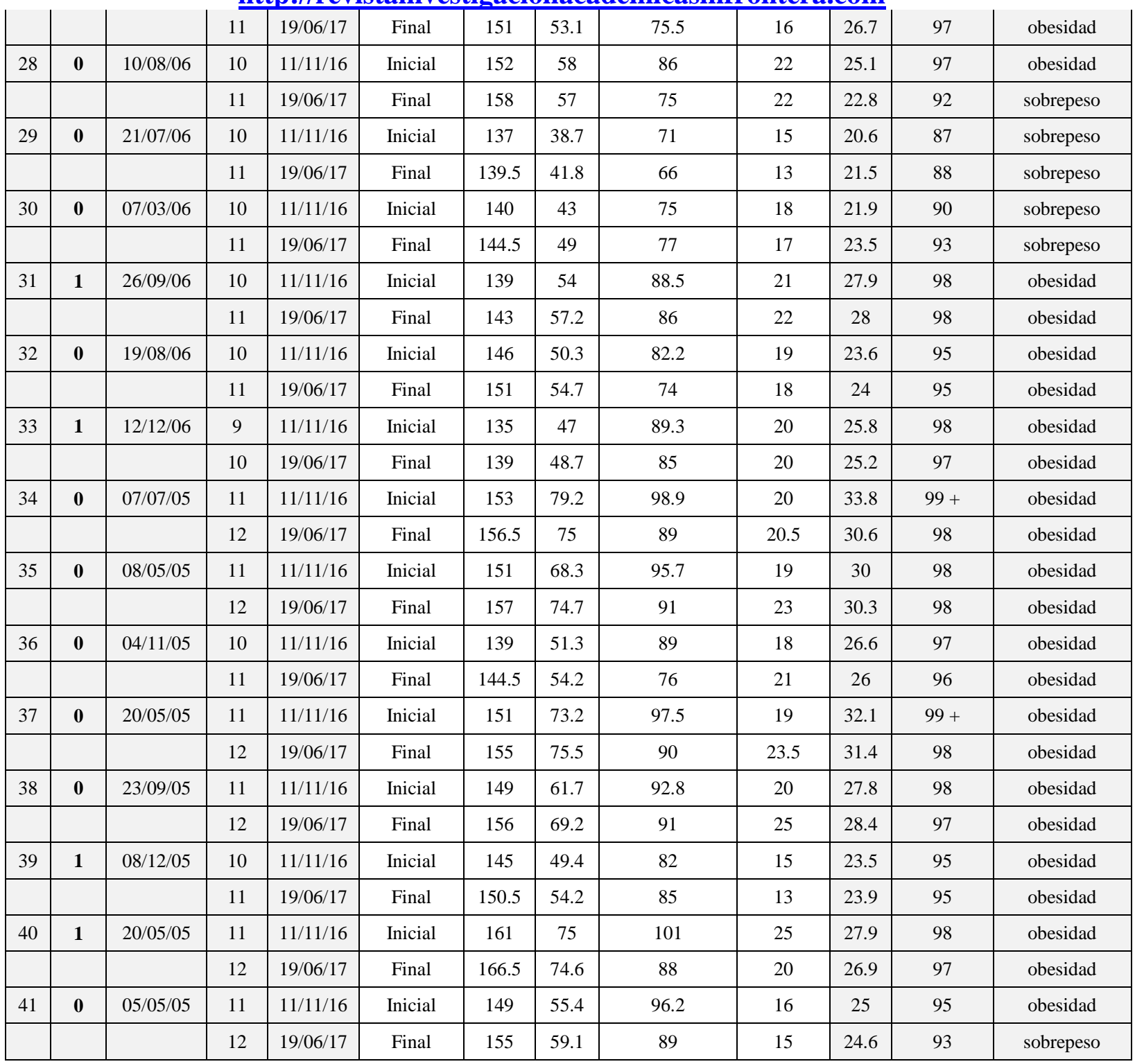

Fuente: Elaboración propia con base en la investigación.

En la Tabla 4 se muestran las pruebas físicas al inicio y al final de su aplicación donde se tomaron en cuenta las variables: 1. Equilibrio (es el número de intentos), donde "0" intentos es lo mejor que se puede lograr, 2. Flexibilidad (flexión del tronco, sentado, piernas extendidas, medido en centímetros), el "0" es neutro lo que no tiene negativo es lo ideal, 3. Salto de longitud pies juntos (es la fuerza de piernas, es un salto sin carrera), es en centímetros y metros, 4. Abdominales (Fuerza 


\section{http://revistainvestigacionacademicasinfrontera.com}

en el abdomen), número de repeticiones y "0" es que no realizo, 5. Velocidad carrera de ida y vuelta(realizar un recorrido 10 veces de $t$ metros), se evalúa en segundos $y$, entre menos segundos realice es lo mejor, y 6. Resistencia (la capacidad del organismo de esforzarse y permanecer activo por largo periodo), el "0" significa que no completo un ciclo.

Tabla 4; Pruebas físicas

\begin{tabular}{|c|c|c|c|c|c|c|c|c|c|c|c|}
\hline No. & Sexo & $\begin{array}{l}\text { Fecha de } \\
\text { nacimiento }\end{array}$ & Edad & Fechas & $\begin{array}{c}\text { Periodo } \\
\text { de } \\
\text { medición }\end{array}$ & Equilibrio & Flexibilidad & Salto & Abdominales & Velocidad & Resistencia \\
\hline 1 & $\mathbf{0}$ & $16 / 08 / 07$ & 9 & $11 / 11 / 16$ & Inicial & 7.0 & -2 & 66.0 & 0.0 & 40.6 & 0 \\
\hline & & & 10 & $19 / 06 / 17$ & Final & 5 & -3 & 85 & 9 & 23.03 & 1.5 \\
\hline 2 & $\mathbf{0}$ & $21 / 11 / 07$ & 8 & $11 / 11 / 16$ & Inicial & 0.0 & -3 & 71.0 & 7.0 & 55.1 & 0 \\
\hline & & & 9 & $19 / 06 / 17$ & Final & 0 & -8 & 80 & 10 & 27 & 0.5 \\
\hline 3 & $\mathbf{0}$ & $31 / 01 / 07$ & 9 & $11 / 11 / 16$ & Inicial & 3.0 & 13 & 110.0 & 15.0 & 41.5 & 1 \\
\hline & & & 10 & $19 / 06 / 17$ & Final & 6 & 15 & 135 & 21 & 21 & 2.5 \\
\hline 4 & $\mathbf{1}$ & $10 / 08 / 07$ & 9 & $11 / 11 / 16$ & Inicial & 9.0 & 5 & 103.0 & 2.0 & 35.23 & 0.5 \\
\hline & & & 10 & $19 / 06 / 17$ & Final & 0 & 7 & 150 & 9 & 17 & 1.5 \\
\hline 5 & $\mathbf{1}$ & $11 / 12 / 07$ & 8 & $11 / 11 / 16$ & Inicial & 0.0 & 2 & 110.0 & 11.0 & 36.9 & 0 \\
\hline & & & 9 & $19 / 06 / 17$ & Final & 9 & -5 & 132 & 27 & 21.44 & 1.0 \\
\hline 6 & $\mathbf{0}$ & $23 / 06 / 07$ & 9 & $11 / 11 / 16$ & Inicial & 7.0 & -9 & 84.0 & 14.0 & 43.1 & 1 \\
\hline & & & 10 & $19 / 06 / 17$ & Final & 5 & -3 & 90 & 17 & 27.43 & 1.5 \\
\hline 7 & $\mathbf{0}$ & $07 / 08 / 07$ & 9 & $11 / 11 / 16$ & Inicial & 0.0 & -7 & 93.0 & 15.0 & 48 & 0 \\
\hline & & & 10 & $19 / 06 / 17$ & Final & 3 & -5 & 120 & 18 & 23.5 & 2.0 \\
\hline 8 & $\mathbf{1}$ & $06 / 07 / 07$ & 9 & $11 / 11 / 16$ & Inicial & 5.0 & 10 & 115.0 & 16.0 & 30.82 & 1 \\
\hline & & & 10 & $19 / 06 / 17$ & Final & 6 & 13 & 142 & 22 & 20.8 & 2.0 \\
\hline 9 & $\mathbf{0}$ & $03 / 08 / 07$ & 9 & $11 / 11 / 16$ & Inicial & 7.0 & 14 & 91.0 & 13.0 & 32.58 & 1 \\
\hline & & & 10 & $19 / 06 / 17$ & Final & 9 & 15 & 114 & 20 & 23.72 & 2.0 \\
\hline 10 & $\mathbf{1}$ & $09 / 11 / 07$ & 8 & $11 / 11 / 16$ & Inicial & 0.0 & 2 & 88.0 & 10.0 & 35.1 & 1 \\
\hline & & & 9 & $19 / 06 / 17$ & Final & 0 & 12 & 100 & 18 & 21.56 & 2.0 \\
\hline 11 & $\mathbf{0}$ & $24 / 04 / 07$ & 9 & $11 / 11 / 16$ & Inicial & 3.0 & 13 & 125.0 & 12.0 & 32.6 & 1 \\
\hline & & & 10 & $19 / 06 / 17$ & Final & 3 & 15 & 123 & 21 & 22.41 & 2.0 \\
\hline 12 & $\mathbf{1}$ & $24 / 09 / 06$ & 10 & $11 / 11 / 16$ & Inicial & 5.0 & 5 & 106.0 & 10.0 & 42.8 & 0 \\
\hline & & & 11 & $19 / 06 / 17$ & Final & 5 & 0 & 120 & 11 & 24.94 & 1.0 \\
\hline 13 & $\mathbf{1}$ & $18 / 06 / 06$ & 10 & $11 / 11 / 16$ & Inicial & 13.0 & 4 & 110.0 & 25.0 & 39 & 0 \\
\hline & & & 11 & $19 / 06 / 17$ & Final & 1 & -3 & 130 & 30 & 22.75 & 5.0 \\
\hline 14 & $\mathbf{1}$ & $10 / 02 / 06$ & 10 & $11 / 11 / 16$ & Inicial & 3.0 & 2 & 88.0 & 14.0 & 41.22 & 0 \\
\hline & & & & & & & & & & & \\
\hline
\end{tabular}




\section{http://revistainvestigacionacademicasinfrontera.com}

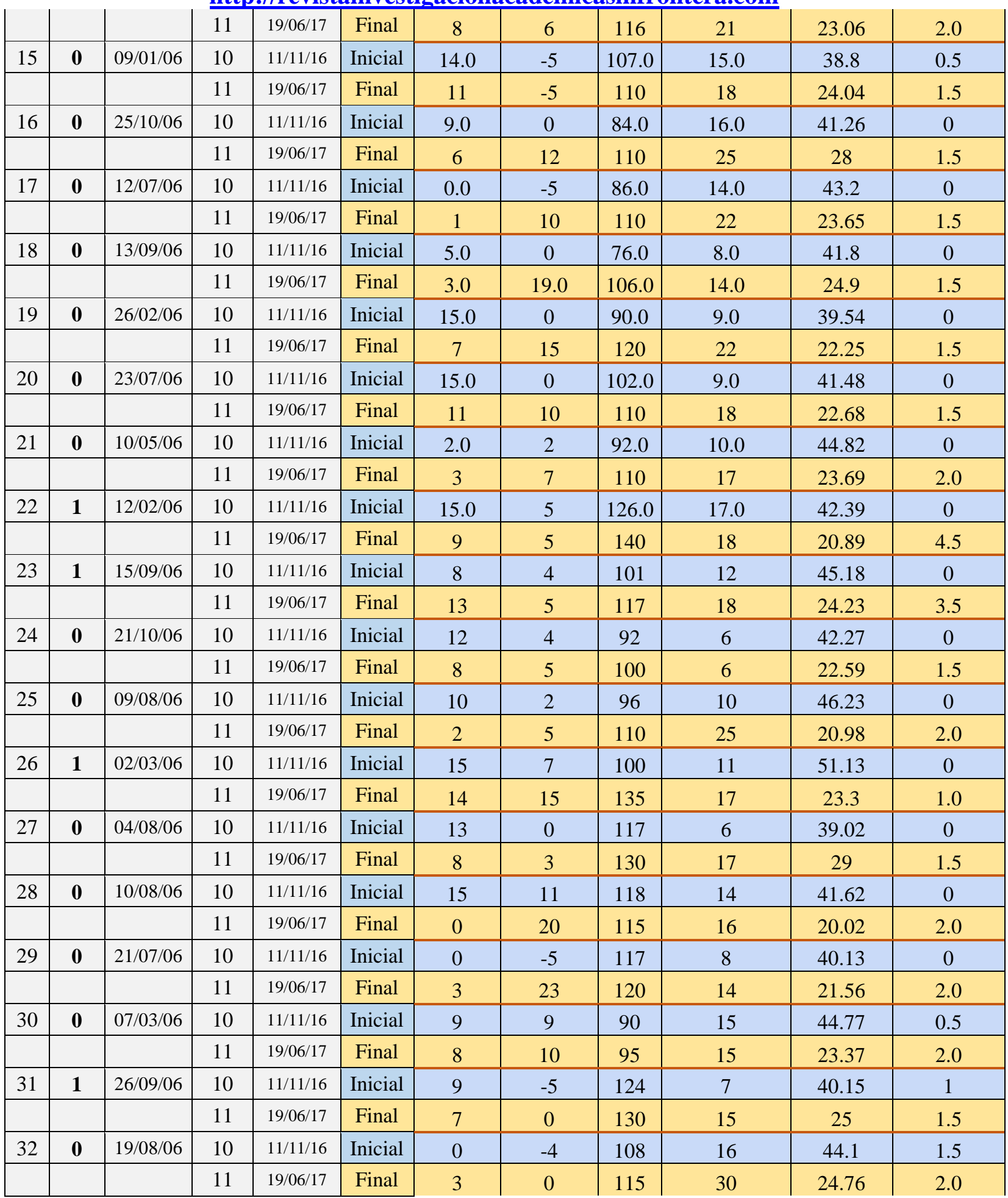




\section{http://revistainvestigacionacademicasinfrontera.com}

\begin{tabular}{|c|c|c|c|c|c|c|c|c|c|c|c|}
33 & $\mathbf{1}$ & $12 / 12 / 06$ & 9 & $11 / 11 / 16$ & Inicial & 2 & -6 & 115 & 13 & 44.7 & 1 \\
\hline & & & 10 & $19 / 06 / 17$ & Final & 0 & 10 & 130 & 18 & 25.08 & 1.5 \\
\hline 34 & $\mathbf{0}$ & $07 / 07 / 05$ & 11 & $11 / 11 / 16$ & Inicial & 0 & 9 & 122 & 18 & 41.6 & 2 \\
\hline & & & 12 & $19 / 06 / 17$ & Final & 0 & 23 & 130 & 28 & 20.3 & 3.0 \\
\hline 35 & $\mathbf{0}$ & $08 / 05 / 05$ & 11 & $11 / 11 / 16$ & Inicial & 3 & 4 & 95 & 10 & 47.6 & 1 \\
\hline & & & 12 & $19 / 06 / 17$ & Final & 2 & 10 & 110 & 20 & 22.5 & 3.0 \\
\hline 36 & $\mathbf{0}$ & $04 / 11 / 05$ & 10 & $11 / 11 / 16$ & Inicial & 6 & 4 & 111 & 11 & 44.4 & 1 \\
\hline & & & 11 & $19 / 06 / 17$ & Final & 6 & 5 & 125 & 22 & 20.61 & 2.0 \\
\hline 37 & $\mathbf{0}$ & $20 / 05 / 05$ & 11 & $11 / 11 / 16$ & Inicial & 0 & 10 & 104 & 16 & 40.78 & 1 \\
\hline & & & 12 & $19 / 06 / 17$ & Final & 0 & 14 & 125 & 25 & 22.05 & 2.5 \\
\hline 38 & $\mathbf{0}$ & $23 / 09 / 05$ & 11 & $11 / 11 / 16$ & Inicial & 12 & 15 & 125 & 15 & 35.6 & 2 \\
\hline & & & 12 & $19 / 06 / 17$ & Final & 7 & 15 & 140 & 25 & 21.6 & 2.5 \\
\hline 39 & $\mathbf{1}$ & $08 / 12 / 05$ & 10 & $11 / 11 / 16$ & Inicial & 2 & 5 & 139 & 30 & 35.63 & 2.5 \\
\hline & & & 11 & $19 / 06 / 17$ & Final & 2 & 7 & 140 & 28 & 27.61 & 5.5 \\
\hline 40 & $\mathbf{1}$ & $20 / 05 / 05$ & 11 & $11 / 11 / 16$ & Inicial & 0 & -10 & 116 & 21 & 31.98 & 0 \\
\hline & & & 12 & $19 / 06 / 17$ & Final & 0 & -3 & 175 & 30 & 19.9 & 1.0 \\
\hline 41 & $\mathbf{0}$ & $05 / 05 / 05$ & 11 & $11 / 11 / 16$ & Inicial & 0 & 13 & 130 & 22 & 38.21 & 1 \\
\hline & & & 12 & $19 / 06 / 17$ & Final & 6 & 15 & 140 & 28 & 22.25 & 2.5 \\
\hline
\end{tabular}

Fuente: Elaboración propia con base en la investigación.

En la misma Tabla 4, se muestra que el desarrollo del programa de Actividad Física realizada durante la investigación mejoró significativamente la motricidad de los niños participantes en el estudio. Los resultados muestran que en la variable 1. Equilibrio, el número de intentos realizado por los niños fueron mejores porque adquirieron fuerza en sus músculos, lo cual les permitió un mejor equilibrio; en la variable 2. Flexibilidad, se puede observar que no se logró en el mismo grado que el resto de las variables, pero si se notó una mejoría significativa considerando su edad; 3. Salto de longitud, se puede observar que de los 41 niños 39 mejoraron la distancia en el salto demostrando la fuerza adquirida en el tronco inferior.; 4. Abdominales, los resultados obtenidos al final de la prueba comparados con el inicio muestran que logran hacer un mayor número de abdominales porque tuvieron más fuerza y pudieron levantarse más continuamente durante las repeticiones; 5. Velocidad, los resultados muestran que el niño inicia de forma lenta y al final se fortalecieron sus piernas y su velocidad mejoró en tiempos y, 6 . 


\section{http://revistainvestigacionacademicasinfrontera.com}

Resistencia, los resultados muestran que la mejor prueba fue realizada en 5.5 ciclos, lo que hace ver una mejor resistencia obtenida en los niños.

Tabla 5: Matriz de correlación de variables de las pruebas físicas

\begin{tabular}{|l|r|r|r|r|r|r|}
\hline & \multicolumn{1}{l}{ Equilibrio } & \multicolumn{1}{c|}{ Flexibilidad } & \multicolumn{1}{c|}{ Salto } & Abdominales & Velocidad & Resistencia \\
\hline Equilibrio & 1 & & & & \\
\hline Flexibilidad & -0.003803007 & 1 & & & \\
\hline Salto & -0.061576376 & 0.325889848 & 1 & & \\
\hline Abdominales & -0.207534849 & 0.269973776 & 0.607874968 & & \\
\hline Velocidad & 0.148531458 & -0.38323767 & -0.540666589 & -0.525884036 & 1 \\
\hline Resistencia & -0.2165801 & 0.355059164 & 0.520515201 & 0.607122023 & -0.66382719 \\
\hline
\end{tabular}

Fuente: Elaboración propia con base en los resultados estadísticos.

La Tabla 5, muestra la matriz de correlación de las variables utilizadas durante las pruebas físicas. El equilibrio muestra que es independiente de las otras variables, para lograr su práctica no influye en el valor de las otras variables, pues, aunque muestra correlaciones negativas, éstas no son significativas, pero el valor de la variable va aumentando a medida que el niño adquiere fuerza en sus músculos. La flexibilidad muestra una relación muy débil con las otras variables, con 0.355, esto se debe posiblemente la flexibilidad tiene poco impacto en la velocidad y la resistencia. En cambio, el salto sí muestra una relación (aunque no muy alta) positiva, es decir los niños que realizaron tienen flexibilidad pueden realizar más fácilmente el salto, los abdominales con mayor velocidad y resistencia.

La variable abdominal, muestra diferencia significativa entre su correlación negativa (0.52) con la velocidad y una correlación positiva de 0.607 con la resistencia. Esto significa que mientras más abdominales practican, su resistencia mejora al esfuerzo, contrario de los niños que realizan velocidad en menos tiempo, su resistencia disminuye. 


\section{http://revistainvestigacionacademicasinfrontera.com}

\section{Conclusiones y propuesta de futuro}

La presencia del sobrepeso y la obesidad entre la población infantil, es un problema que va en aumento en países de todo el mundo. Su desarrollo obedece a factores de tipo genético y a aquellos relacionados con el estilo de vida, entre los que se destaca la actividad física. En la actualidad, entre los obstáculos para que los niños realicen ejercicio, se señalan distractores como el uso de la televisión, videojuegos, computadoras y, el medio ambiente en el que se desarrollan ha sustituido el juego al aire libre, fomentando por su parte, el ocio sedentario, que es cada vez más común. Hay estudios en los que se resalta que estos comportamientos se acentúan durante el fin de semana. También se menciona que el comportamiento sedentario es más frecuente en niñas que en los niños, debido al tipo de juegos que prefieren (Trejo et al., 2012; Habib-Mourad, 2014).

Los resultados del presente estudio demostraron que la implementación de un programa de actividad física en niños con sobrepeso y obesidad, les permitió obtener una mejoría muy significativa en sus capacidades física condicionales y coordinativas. Los niños no presentaron una disminución en su peso porque se puede ver que el niño aumento en su talla o estatura. Lo anterior, demuestra que se cumplió con el objetivo planteado en la investigación demostrando que el ejercicio físico es un recurso de gran importancia para el tratamiento y prevención del sobrepeso y de la obesidad, por lo que es necesario de implementar un programa de actividad física basada en el desarrollo de capacidades físicas condicionales y coordinativas a través de ejercicios aeróbicos y anaeróbicos como hábito saludable para el control de sobrepeso y obesidad infantil.

Teniendo en cuenta este panorama, se puede concluir que es necesario por lo tanto "reintroducir" la actividad física en la vida de los niños asociándola con la recreación, propiciando patrones desde la niñez importantes para cimentar los hábitos de actividad física en el futuro. La 


\section{http://revistainvestigacionacademicasinfrontera.com}

escuela por lo tanto tiene la obligación de establecer programas de actividad física para niños con sobrepeso y obesidad, en el horario extraescolar de escuelas de tiempo completo.

Es importante destacar que la infancia es un momento clave para generar conductas de salud, por lo que es importante desarrollar programas de intervención en esta etapa que permitan aumentar el tiempo que se dedique a la actividad física junto a la obtención de conocimientos nutricionales, para ello el contexto político y social deberá ser favorable, y deberán estar basados en intervenciones anteriores que hayan obtenido resultados positivos, logrando su mantenimiento a largo plazo, para poder reducir la prevalencia de obesidad y conseguir que la población infantil crezca sana y activa.

Este estudio presenta varias limitaciones que sugieren vías de investigación futuras, donde deberían profundizar en variables que impactan directamente en la generación del desarrollo motor y las causas de la malnutrición por exceso en los niños. Es importante profundizar más en una idea más clara de la relación y posibles efectos del sedentarismo y obesidad infantil en el desarrollo psicomotor infantil nos permitirá tener una visión más integradora de todos los factores que pueden afectar al desarrollo psicomotriz normal de niños y niñas y así poder tomar medidas y estrategias adecuadas de prevención, control o intervención en los plazos más eficaces para que todos los niños tengan la posibilidad de mejorar sus habilidades y rendimiento psicomotor.

\section{Referencias bibliográficas}

Bloomgarden, Z.T. (2004). Tipe 2 diabetes in young: the evolving epidemic. Diabetes Care; Vol. 27, pp. $998-1010$

Bucco L, Zubiaur M. (2013). Desarrollo de las habilidades motoras fundamentales en función del sexo y del índice de masa corporal en escolares. Cuad. Psicol. del Deport;13(2), pp. 63-72. 


\section{http://revistainvestigacionacademicasinfrontera.com}

Ceballos, O., Álvarez, J., Torres, A. y Zaragoza, J. (2009). Actividad física y calidad de vida. Monterrey: Publicaciones UANL.

Crovetto, M., Zamorano, N. y Medinelli A. (2010). Estado nutricional, conocimientos y conductas en escolares de kinder y primer año básico en 3 escuelas focalizadas por obesidad infantil en la comuna de Valparaiso, Chile. Rev. Chil. Nutr;37:309-20.

Deurenberg, P., Weststrate, J.A. y Seidell, J.C. (1991). Body mass index as a measure of body fatness: age and sex speci c prediction formulas. Br. J. Nutr, 65, pp. 105-114.

Downward, P. y Riordan, J. (2007). Social Interactions and the Demand for Sport: An Economic Analysis. Contemporary Economic Policy. Vol. 25, núm. 4.

Eberth, B. y M. Smith (2010). Modeling the Participation Decision and Duration of Sporting Activity in Scotland. Economic Modeling. Vol. 27, núm. 4.

Elena A. (2006). El aprendizaje motriz en los primeros tres años de vida del niño. Pensam. Educ.; Vol. 38: pp. 218-30.

Figueroa, D. (2009). Obesidad y Pobreza: marco conceptual para su análisis en Latinoamérica, Saúde Soc. São Paulo, 18, (1), pp. 103-117.

Garita E. (2006) Motivos de participación y satisfacción en la actividad física, el ejercicio físico y el deporte. Revista MHSalud® (ISSN: 1659-097X) Vol. 3. № 1.

Habib-Mourad C, Moore H, Nabhani-Zeidan M, Hwalla N, Summerbell C. Educ Health. (2014). Health-E-PALS: promoting Healthy Eating and Physical Activity in Lebanese school children - Intervention development.

Humprhreys, B. y J. Ruseski (2007). Participation in Physical Activity and Government Spending on Parks and Recreation. Contemporary Economic Policy. Vol. 25, núm. 4.

Kipping RR, Jago R, Lawlor DA. Obesity in children. Part 1: Epidemiology, measurement, risk factors, and screening. BJM.2008; Vol. 337: pp. 922-27. 


\section{http://revistainvestigacionacademicasinfrontera.com}

Kwame, N. (2009). An Exploration of Demand for Physical Activity. Tesis de doctorado, United Kingdom, Health Economic Research Group, Brunel University.

Lera, F., García, J. y Suárez, M. (2009). Estimation of a Structural Model of the Determinants of the Time Spent on Physical Activity and Sport: Evidence for Spain" Economic Discussion Papers. España, Departamento de Economía, Universidad de Oviedo.

Mercado, P. y Vilchis, G. (2013). La obesidad infantil en México. Alternativas en Psicología. Revista Semestral. Tercera Época. Año XVII. Número 28, pp. 49-57.

Moreno LA, Rodríguez G, Fleta J, Bueno-Lozano M, Lazaro A, Bueno G. (2010). Trends of dietary habits in adolescents. Crit Rev Food Sci Nutr. 50 (2), pp.106-12.

Moreno, M. (2012). Definición y clasificación de la obesidad. Revista Médica Clínica CONDES; 23(2); pp. 124-128.

Nielsen, G.A., Andersen, L.B. (2003). The association between high blood pressure, physical fitness, and body mass index. Prev Med; Vol. 36: pp. 229-234.

Nigg, C. R. (2003). Do sport participation motivations add to the ability of the transtheoretical model to explain adolescent exercise behavior? International Journal of Sport Psychology, Vol. 34, pp. 208- 225.

O.M.S. (2009). Global health risks: Mortality and burden of disease attributable to selected major risks. Geneva: World Health Organization.

Onis, M., Blössner, M., \& Borghi, E. (2010). Global prevalence and trends of overweight and obesity among preschool children. American Journal of Clinical Nutrition, Vol. 92(5), pp. 1257-1264.

Organización Mundial de la Salud. (2012/6). Obesidad y sobrepeso. Recuperado el 16 de Octubre, 2017, de: http://www.who. int/mediacentre/factsheets/fs311/es/

Parsons, T.J., Manor O, Power C. (2006). Physical activity and change in body mass index from adolescence to mid-adulthood in the 1958 British cohort. Int J Epidemiol; Vol. 35: pp.197204.

Peña, M. y Bacallao, J. (2001). La obesidad y sus tendencias en la Región. Revista Panamericana de Salud Pública, Vol. 10(2), pp. 45-78. 


\section{http://revistainvestigacionacademicasinfrontera.com}

Quizán Plata, T., Álvarez Hernández G. y Espinoza López, A. (2007). Obesidad infantil: el poder de la alimentación y la actividad física. Revista Universidad de Sonora. Recuperado el 16 de Octubre, 2017, en: http://www.revistauniversidad.uson.mx/revistas/22-22articulo\%203.pdf

Ramírez, W, Vinaccia S. y Suárez, G.R. (2004). Impacto de la actividad física y el deporte sobre la salud, la cognición, la socialización y el rendimiento académico; una revisión teórica. Revista de Estudios Sociales, Vol. 18, pp. 67-75.

Rodríguez Martín, Amelia, Novalbos Ruiz, José P., Villagran Pérez, Sergio, Martínez Nieto, José M., \& Lechuga Campoy, José L. (2012). La percepción del sobrepeso y la obesidad infantil por parte de los progenitores. Revista Española de Salud Pública, Vol. 86(5), pp. 483-494.

Rodriguez-R A. (2006). La obesidad infantil y los efectos de los medios electrónicos de comunicación. Medigraphic Artemisa en linea.Vol. 8, pp. 95-8.

Salud, O. M. (2012). Encuesta Nacional de Salud y Nutrición. 2012. SS. Organización Mundial de la Salud. Obtenido de la Organización Mundial de la Salud: http://www.who.int/dietphysicalactivity/factsheet_young_people/es/

Sallis, J. Owen, N. (1999). Physical activity and behavioral medicine. Sage publications. California, pp.110-133.

SEEDO (2013). La obesidad, ahora sí una enfermedad de peso. Recuperado el 6 de septiembre, 2017, de http://www.seedo. es/index.php/la-obesidad-ahora-si-una-enfermedad-depeso.

Strong, W.B., Malina, R.M., Blimkie, C.T., Daniels, S.R., Dishman,, R.K. y Gutin, B. (2005). Evidence based physical activity for school-age youth. Pediatric. Vol. 146, pp. 732-737.

Trejo Ortiz, Perla María, Jasso Chairez, Susana, Mollinedo Montaño, Fabiana Esther, \& Lugo Balderas, Lilia Guadalupe. (2012). Relación entre actividad física y obesidad en escolares. Revista Cubana de Medicina General Integral, 28(1), pp. 34-41.

Vicente-Rodríguez, G., \& Benito, P., \& Casajús, J., \& Ara, I., \& Aznar, S., \& Castillo, M., \& Dorado, C., \& González-Agüero, A., \& González-Gallego, J., \& González-Gross, M., \& GraciaMarco, L., \& Gutiérrez, Á., \& Gusi, N., \& Jiménez-Pavón, D., \& Lucía, A., \& Márquez, S., \& 
Núm. 26

ISSN: 2007-8870

\section{http://revistainvestigacionacademicasinfrontera.com}

Moreno, L., \& Ortega, F., \& de Paz, J., \& Ruiz, J., \& Serrano, J., \& Tur, J., \& Valtueña, J. (2016). Actividad física, ejercicio y deporte en la lucha contra la obesidad infantil y juvenil. Nutrición Hospitalaria, 33 (9), pp. 1-21. 